\title{
A Challenge to the New Metaphysics: deRosset, Priority, and Explanation
}

\author{
David Fisher, Hao Hong, Timothy Perrine
}

\author{
Penultimate Draft. Please cite published version. \\ Forthcoming in Synthese, DOI: 10.1007/s11229-019-02468-3
}

\begin{abstract}
Priority Theory is an increasingly popular view in metaphysics. By seeing metaphysical questions as primarily concerned with what explains what, instead of merely what exists, it promises not only an interesting approach to traditional metaphysical issues but the resolution of some outstanding disputes. In a recent paper, Louis deRosset argues that Priority Theory isn't up to the task: Priority Theory is committed to there being explanations that violate a formal constraint on any adequate explanation. This paper critically examines deRosset's challenge to Priority Theory. We argue that deRosset's challenge ultimately fails: his proposed constraint on explanation is neither well-motivated nor a general constraint. Nonetheless, lurking behind his criticism is a deep problem for prominent ways of developing Priority Theory, a problem which we develop.
\end{abstract}

\section{Introduction}

On a Quinean view, the question of ontology is the question of what there is (cf. Quine (1948)). But there are different ways of answering this question. One camp answers this question by drawing on ordinary and scientific theorizing, thereby claiming that there are many different ordinary objectstables, chairs, baseballs, tectonic plates, rain drops, feathers, etc. This broad camp, though, faces problems of explanation - in order to explain why the window shattered, must we speak of a baseball? Surely there merely being particles arranged in a certain way would suffice. To postulate the existence of a further thing - the baseball - would be to postulate an unnecessary entity that did no explanatory work (cf. Merricks (2007)). An alternative camp-the narrow camp-urges that we answer this question by quantifying over very little, perhaps just simples (or, if we are feeling generous, organisms too; cf. van Inwagen (1990) and Merricks (2007)). But the narrow camp faces linguistic and epistemological problems: it seems to entail that much of our ordinary and scientific discourse - which is existentially permissive - is false. ${ }^{1}$ The epistemological problem is that the best evidence we have for what there is comes from ordinary and scientific contexts; the narrow camp seems to ask us to turn our backs on that evidence (cf. Kelly (2008)).

The dialectic between these two camps is well-known and entrenched. Here the Priority Theorist promises a solution. She claims that she can retain the wide range of existence claims the broad camp endorses, while respecting the restricted explanatory claims of the narrow camp thereby combining the best of both. She does this by abandoning the Quinean view of ontology. The

\footnotetext{
${ }^{1}$ van Inwagen (1990) denies this is a consequence of his view. For critical discussion, see O'Leary-Hawthorne \& Michael (1996) and Fisher (Forthcoming).
} 
question of ontology is not primarily a question about what there is but what grounds or explains what. Specifically, ontology is a matter of identifying the "fundamental" ground of things. The Priority Theorist thus advocates a reorientation of our metaphysical theorizing. ${ }^{2}$

Priority Theory is an important view in contemporary metaphysics. First, it promises an irenic solution to some well-known disputes. Second, it offers an important and contrasting approach to the Quinean view that has dominated philosophy for many decades - an approach that may be, as Schaffer (2009) suggests, more true to the historical task of ontology than the Quinean view. Finally, it postulates important kinds of relations - explanatory or grounding relations - for the metaphysician to explore and work with. It is no wonder that more and more contemporary metaphysicians are drawn to this approach to ontology. But, we think, the initial novelty period of Priority Theory is now past, and to make progress we must turn to arguments for and against the position.

Our aim here is to evaluate a recent objection to Priority Theory made by Louis deRosset (2010). deRosset objects that Priority Theory is unpromising because its proposed explanations violate an adequacy constraint on explanations he calls the Determination Constraint. We argue that deRosset's objection fails: his proposed constraint is neither well-motivated nor general. But we distill from deRosset's objection a general structure for objecting to Priorty Theory: given certain adequacy constraints on Priority Theory explanations, it will derive a contradiction given one uncontroversial assumption. In the remainder of the paper, we develop an objection to Priority Theory using this general structure. We first articulate a proposed constraint on Priority Theory, namely, that for any facts it intends to explain, there is a set of statements that do not mention the objects in those facts but nonetheless implies that those facts obtain. We then show how, assuming Priority Theory, when this constraint is applied to an important class of facts which ought to have explanations, it implies a contradiction. We show how several ways of articulating or developing Priority Theory fail to avoid this problem. The upshot is that while Priority Theory may weather deRosset's challenge, it remains to be seen whether it can be developed in a way that is not only coherent but plausible.

We do this in four sections. In $\S 2$, we lay out Priority Theory and deRosset's criticism of it. In $\S 3$, we argue that deRosset's argument for the Determination Constraint is implausible and that the constraint is too similar to an outdated model of explanation. Finally, in $\S 4$, we develop a problem for Priority Theory: given a certain constraint on its explanations a contradiction will be derivable given the uncontroversial assumption that a certain kind of fact has an explanation. We then show how several, increasingly complex ways of articulating Priority Theory imply this constraint and so encounter this problem. We conclude by briefly discussing how difficult it will be for proponents of Priority Theory to avoid this problem.

\footnotetext{
${ }^{2}$ Schaffer (2009) is a position paper advocating such a reorientation. Much of the recent enthusiasm for 'grounding' takes the form of advocating a Priority Theoretic reorientation of ontological theorizing. Cf. Bliss \& Trogdon (2014) and the citations therein. So does enthusiasm for the distinct, but related, concept of fundamentality. Indeed, deRosset probably intends the label 'Priority Theory' as just a covering term for these different reorientations currently in vogue. Note that Priority Theory is more than just the claim that there are non-causal explanations, a claim probably no one ever questioned. It is rather the claim that certain non-causal explanations or explanatory relations are primarily what ontologists should be studying. (This is consistent with ontology, and metaphysics more generally, also being concerned with other questions, like existence questions. After all, existence facts are presumably quite relevant to explanatory facts.)
} 


\section{Exposition of deRosset's Argument}

\section{1 deRosset's Formulation of Priority Theory}

Unlike the Quinean, for the Priority theorist the central issue for ontology is not what we quantify over but what explains what. Following deRosset, let's call a fact fundamental if it is not explained by any other fact (with a nonfundamental fact being one that isn't fundamental). Call an entity or kind fundamental if "reference to it must be made in any complete statement of all the fundamental facts" (2010: 75); that is, an entity is fundamental if it figures in some fundamental fact. ${ }^{3}$ As deRosset puts it, the Priority Theorist then accepts:

(Sparsity) The ontological sparsity of the world is determined by the number and variety of fundamental entities and kinds. (2010: 75)

Nevertheless, the Priority Theorist tries to retain the motivation of the broad Quinean camp by accepting:

(Modesty) The claims of common sense abetted by science about the existence and features of macroscopic concreta are roughly correct: there are tables, raindrops, tectonic plates, galaxies, etc. (2010: 75$)$

But, like the narrow camp, she does not see these entities as being explanatory. Rather, she thinks:

(Explanation) The existence and features of the macroscopic concrete objects alleged by common sense abetted by science can be completely explained solely by reference to the existence and properties of other things. (2010: 75)

Given our definitions of 'fundamental', (Explanation) implies:

(Priority) The fundamental facts do not include any distributions of features over ordinary macroscopic concreta, including tables, raindrops, tectonic plates, galaxies, and the like. (2010: 81)

After all, suppose some fundamental fact referred to (e.g.) a table. Then given the definition of fundamental fact, it would follow that this fact is not explained by any other fact. But this is inconsistent with (Explanation) which holds that facts about tables can be explained.

Following deRosset, we'll take these theses to define Priority Theory. ${ }^{4}$

\section{2 deRosset on Adequate Explanations}

deRosset's criticism of Priority Theory is that its proposed explanations violate an adequacy constraint on explanations, what he calls the Determination Constraint. He arrives at this constraint by generalizing from what he takes would be defects for certain (purported) scientific explanations. Some toy explanations he lists:

\footnotetext{
${ }^{3}$ We'll simply treat facts as true propositions, for reasons of simplicity. Nothing will turn on this. We will also be loose with use-mention throughout, as our intentions should make our meaning clear.

${ }^{4}$ These theses are consistent with the fundamental objects being microscopic things or the whole universe. (The latter position is sometimes referred to as Priority Monism.) Other positions might be consistent with these theses but sit poorly with their motivation. For instance, composition as identity is perhaps best seen as a way to thread the needle between the broad and narrow camps by saying that the existence of (e.g.) baseballs just is the existence of certain microscopic particles organized a certain way. (For more on composition as identity, see Wallace (2011a,b).) We think endorsing Priority Theory would alleviate some (though maybe not all) of the motivation for such a position.
} 
A given isotope of gold has a certain atomic mass in virtue of containing a certain number of protons and neutrons. Ethanol is miscible in water in virtue of containing a hydroxide group. Diamond is hard because each carbon atom in its crystalline structure is bonded to each of its neighbors. (2010: 78)

We might model these kinds of explanations as follows. Suppose we offer an explanation of an atomic fact, something of the form $r$ is $F$ - that some particular raindrop $(r)$ is translucent $(F)$, for instance. ${ }^{5}$ He claims "... there's something wrong with or missing from an explanation of $r$ 's being $F$ if there is a situation in which something is just like $r$ so far as the explanans goes, but lacks $F$ " (2010: 79). ${ }^{6}$ deRosset's comments here suggest a requirement for adequate explanations, what we'll call the Likeness Principle:

(LP) If an explanation of some fact about $r$ is adequate then anything that is like $r$ as far as the explanans goes should be like $r$ as far as the explanandum goes.

To use his own example, consider the claim that some diamond $d$ is hard because its constituent molecules are arranged in a particular crystalline structure. If some other mineral, $d^{*}$, were like $d$ as far as the explanans goes - its constituent molecules were also so arranged - but it were not like $d$ as far as the explanandum goes - it wasn't hard - then the proposed explanation would be inadequate.

deRosset arrives at his constraint on adequate explanations by, first, regimenting the form of explanatory proposals generally, then, second, explicating (LP) in light of that general form. First: he proposes to regiment relevant explanatory proposals as taking the form:

(Prop) $r$ is $F$ because $\phi(r, \vec{a}) .^{7}$

"where all of the individuals involved in the explanans are denoted by exactly one term among $r$ and $\vec{a}$ and $\phi$ says how the properties and relations used for the explanation are distributed over those individuals' (2010: 78). E.g., raindrop $r$ is tear-shaped $(G)$ because $r$ is composed of molecules $(\vec{a})$ which bear certain spatial relations to each other, with $\phi(r, \vec{a})$ standing in for a specification of the exact spatial relations among the $\vec{a}$.

A quick but important digression on notation. $\phi(r, \vec{a})$ is notation familiar from mathematical logic. Generally speaking, $\phi(r, \vec{a})$ acts as function from terms, here $r$ and $\vec{a}$, to formulas of an assumed object language. The metalinguistic expression, ' $\phi(r, \vec{a})$ ' serves as a display for object language sentences that can be useful in certain ways. For instance, one can use this function to help formulate the familiar rule of Existential Generalization like this:

- From $\phi(c)$ infer $(\exists x) \phi(x)^{8}$

But there are some fine points about how exactly that function is defined that logicians differ over. Further, the details of deRosset's arguments are sensitive to these fine points. Specifically, deRosset's employment of the notation in his regimentation, (Prop), builds in two conditions not

\footnotetext{
${ }^{5}$ We switch from a generic facts, like those used in deRosset's quote, to an atomic fact because deRosset scrutinizes atomic facts the most.

${ }^{6}$ By 'situation' deRosset has in mind some actual situation and not a merely possible one. (Some critics, e.g. Skiles (2015), have not been sensitive to this.) But we'll follow him in this here.

${ }^{7}$ To reduce clutter, we have rewritten finite sequences of terms $a_{1}, \ldots, a_{n}$ in vector notation, $\vec{a}$, in quotations and throughout. In what follows, lower case Latin letters late in the alphabet, $x, y, \ldots$ will be reserved for variables, and any other lower case Latin letters for constants. A vector $\vec{x}$ (resp. $\vec{a}$ ) will be assumed to be a sequence of variables (resp. constants) only - there won't be need for "mixed" vectors.

${ }^{8}$ Often logicians use notation like $(\exists x) \phi(c / x)$ to indicate that $x$ is being substituted for $c$ in the formula $\phi(c)$. We follow deRosset in leaving this implicit.
} 
every logician imposes (cf. Marker (2002: 11)) but which also have some precedent (cf. Mendelson (1979:48)). First, he stipulates every term that appears in the object language sentence denoted must show up in the display, hence that every term in the sentence denoted by $\phi(r, \vec{a})$ must be either $r$ or one of $\vec{a}$. Second, the display is permitted to contain too many terms: that is, he allows that some of the terms among $r$ and $\vec{a}$ might not actually be in the object language sentence. So it is consistent with this notation that, e.g., $\phi(r, \vec{a})$ denotes $H a(2010: 79 \mathrm{fn} .25){ }^{9}$ This second condition may feel slightly misleading - especially if we naturally, but mistakenly, read $\phi(r, \vec{a})$ as kind of placeholder for a (complex) object language predicate taking $r$ and $\vec{a}$ as arguments. (After all, if $r$ doesn't occur in the denoted sentence then it's not an argument of any predicate of the sentence.) Be that as it may, the notation is perfectly well-defined and, as we mentioned, not unprecedented. In any case, as we'll see in the next section, understanding the details of deRosset's argument requires understanding this fine point about the notation he uses in giving his argument.

Returning to the thread, (LP) tells us that a proposed explanans of $r$ 's being $F$ is inadequate if two "likeness" facts obtain: there is an object, $r^{*}$, which is like $r$ so far as the explanandum goes but unlike $r$ so for as the explanans goes. deRosset proposes that we explicate these ideas of "likeness" in terms of the Tarskian notion of satisfaction. ${ }^{10}$ Thus, on his explication, $r^{*}$ is $u n$ like $r$ so far as the explanandum goes only if $r$ satisfies ' $x$ is $F$ ' and $r^{*}$ fails to satisfy it. Likewise, $r^{*}$ is like $r$ so far as the explanans goes only if each satisfies $\phi$ along with some $\vec{b}$. (Following Field (1974), when something satisfies $\phi$ along with some vector, we will say it "partially satisfies" $\phi$.) In this way, deRosset explicates the (LP)'s talk of "likeness" into talk of satisfaction and partial satisfaction. Introducing the technical term 'confounding case,' deRosset says that an explanatory proposal has a confounding case if there is an object that partially satisfies the relevant explanans of the explanatory proposal, while failing to satisfy the explanandum of that proposal (2010: 80). Returning to the diamond example, then, the original proposed explanation in canonical form might be:

\section{$\operatorname{Hard}(d)$ because Compose $(\vec{a}, d) \wedge$ Crystalline-Structure $(\vec{a})$.}

Under the recent explication, there would be a "confounding case" for this proposal, and thus a violation of (LP) so understood, if there were some $d^{*}$ and $\vec{b}$ such that

$$
\text { Compose }\left(\vec{b}, d^{*}\right) \wedge \text { Crystalline-Structure }(\vec{b}) \wedge \neg \operatorname{Hard}\left(d^{*}\right) \text {. }
$$

In what follows, we will follow deRosset in using the term 'confounding case' as he defines it in terms of satisfaction.

Given both the claim that explanatory proposals can be adequately regimented through (Prop) and the claim that (LP)'s talk of "likeness" can be adequately explicated in terms of satisfaction, deRosset arrives at his proposed constraint on explanation, which he calls the Determination Constraint:

(Det) An explanatory proposal is adequate only if there is no confounding case for it. (2010: 80)

(where the term 'confounding case' is defined above in terms of satisfaction). Whether such claimsand by extension (Det) - are warranted is something we address below.

\footnotetext{
${ }^{9}$ This is harmless in many applications. For instance, if $c$ doesn't actually occur in $\phi(c)$ then the $\exists x$ in $\exists x . \phi(x)$ will just be a vacuous quantifier. Although deRosset doesn't make this explicit, the reason for this second condition is that it allows for a syntactically clean generalization over all explanatory proposals à la (Prop). Were we to instead require that $r$ must in occur in the object language sentence denoted by $\phi(r, \vec{a})$ then the kind of explanations Priority Theorists seek to produce would not fit the form of (Prop) — as, by definition, a nonfundamental raindrop $r$ may not occur in the explanans of some explanation of its being $F$. And thus (Prop) would not a general form of explanation.

${ }^{10}$ Tarski (1983) defined satisfaction as one step removed from the truth: a term or sequence of terms satisfies a formula just if subbing that term (sequence) into the formula results in something true.
} 


\section{3 deRosset's Argument}

deRosset's main argument against Priority Theory can be put like this:

(P1) If Priority Theory is true, then there are adequate explanations that violate the Determination Constraint.

(P2) No adequate explanation violates the Determination Constraint.

$\therefore$ Priority Theory is false.

His positive argument for $\mathrm{P} 2$ primarily consists in his explication of the (LP) - explained above plus replies (2010: 85-88) to some objections. ${ }^{11}$ His first premise, P1, is the more controversial, and is less obvious. deRosset nevertheless provides a seemingly compelling argument for it. He defends P1 by generalizing from a toy explanation that, while simplified, is illustrative of many explanations the Priority Theorist might give, showing how confounding cases can be easily generated for such explanations. We'll first go through the case in an intuitive way; then, to show how minimal the assumptions are, we'll work through it in a way that is more abstract but more logically perspicuous.

Consider two facts that the Priority Theorist accepts given her acceptance of (Modesty):

(Drop) $r$ is translucent. (For $r$ some particular raindrop.)

(Plate) $p$ is not translucent. (For $p$ some particular tectonic plate.)

According to (Explanation), both (Drop) and (Plate) have adequate explanations solely in terms of fundamental facts. deRosset's key claim is that any explanation the Priority Theorist can give of (Drop) will have a confounding case in (Plate). But if an explanation has a confounding case, by definition it violates the Determination Constraint, which suffices for P1.

Here is how (Plate) furnishes a confounding case, in deRosset's technical sense, for any explanation of (Drop) a Priority Theorist might give. Recall that for the Priority Theorist there must be an explanation for (Drop) that does not refer to the raindrop $r$ at all in its explanans. (We assume raindrops are not fundamental.) In accordance with (Prop), that explanation will have the form ' $r$ is translucent because $\phi(r, \vec{a})^{\prime}$ ', where none of terms occurring in $\phi(r, \vec{a})$ denote that raindrop. Hence, in particular, $r$ cannot occur in the sentence $\phi(r, \vec{a})$, which is consistent with how deRosset understands this notation.

Since by assumption $r$ doesn't actually occur in the original explanans, $\phi(r, \vec{a})$, the result of "replacing" $r$ with $p$ in the explanans will be the exact same sentence. It is the "null replacement," if you like. (Compare: if there are no elephants in a room, the result of replacing all of the elephants with hippos will leave the room unchanged.) Thus, given that $r$ doesn't occur in the explanans, the object language sentences denoted respectively by $\phi(r, \vec{a})$ and by $\phi(p, \vec{a})$ are one and the same (cf. deRosset (2010: $82 \mathrm{fn} .29)$ ). Since the explanans $\phi(r, \vec{a})$ is identical with $\phi(p, \vec{a})$, they will of course agree in terms of what satisfies them. But $r$ satisfies the explanans $\phi(r, \vec{a})$. Thus, $p$ also satisfies it. But clearly $p$, being a tectonic plate, is not translucent. Thus $p$ does not satisfy the explanandum of $r$ 's being translucent, despite satisfying the explanans of $r$ 's being translucent. But that is just what it is for something to be a confounding case. So Plate will be a confounding case for any explanation of Drop that the Priority Theorist might give; P1 of deRosset's argument is thus true.

\footnotetext{
${ }^{11}$ We represent deRosset as maintaining that (Det) is a general constraint, but his text is a little ambiguous. His discussion of (Det) doesn't explicitly include a relativization to a certain kind of explanation. But the prefactory comments to (Det) suggest that he is interested in non-causal explanations, that is, explanations where the explanans is not a cause of the explanandum. This more restrictive understanding of (Det) will not matter for our argumentation below.
} 
This argument did not really substantively depend upon the fact that we focused on a particular raindrop, tectonic plate, and the predicate 'translucent'. In fact, the argument for P1 turns on very few assumptions, as we can see by working through a more abstract version of the argument.

To show that P1 is true, let us assume for reductio that both Priority Theory and (Det) are true. Priority Theory implies (Modesty), which requires

(1) $r$ is $F$

where $r$ is nonfundamental and not everything is $F$. (If not everything is $F$ we'll say $F$ is "nontotal".) This is a commitment of (Modesty) because, after all, ordinary life and scientific theorizing do not claim that everything has all the same properties! Given the regimentation (Prop) and (Explanation), it follows that (1) has an adequate explanation, something of the form

(2) $r$ is $F$ because $\phi(r, \vec{a})$

for some $\vec{a}$, where $r$ doesn't occur in $\phi(r, \vec{a})$. Now a confounding case for $(2)$ would be some $r^{*}$ and some $\vec{b}$ such that

(3) $\phi\left(r^{*}, \vec{b}\right) \wedge \neg F r^{*}$

is true. Having assumed (Det), if (2) is adequate it would lack any confounding case - nothing of the form (3) would be true. In other words, if (2) and (Det) are true, so is:

(4) $\neg(\exists x, \vec{y})[\phi(x, \vec{y}) \wedge \neg F x]$

((4) is just the generalization of (3) that is then negated.) But since $r$ doesn't occur in the explanans $\phi(r, \vec{a})$ in $(2),(4)$ implies

(5) $(\exists \vec{y})[\phi(x, \vec{y})] \rightarrow(\forall x)(F x) .{ }^{12}$

Returning to (2), given the factivity of explanations, it will be the case that $\phi(r, \vec{a})$, which implies the antecedent of $(5)$ by ( $\exists$-instantiation). And modus ponens gives us

(6) $(\forall x)(F x)$.

- that everything is $F$, which contradicts the fact that $F$ is non-total. Thus, our assumption-i.e. that (Det) is true, that no explanation has a confounding case - is false. Thus, if Priority Theory is true, then (Det) is false, which is just (P1). By inspection we can see that argument relies only on theses of Priority Theory, (Modesty) and (Explanation), along with (Det) and deRosset's definition of 'confounding case'.

So goes deRosset's argument against Priority Theory. We don't deny that his argument seems somehow fishy. Nevertheless, the main argument is deductively valid, so that if the argument fails, one of the premises must go. The trick is to explain which and why exactly it is false. This will be the task of $\S 3$.

\footnotetext{
${ }^{12}$ In fact (4) and (5) are logically equivalent. Proof sketch: (4) and

$\left(4^{\prime}\right)(\forall x, \vec{y})[\phi(x, \vec{y}) \rightarrow F x]$
}

are equivalent by the usual quantifier exchange rules and facts about the sentential connectives. But $\left(4^{\prime}\right)$ is the prenex normal form of (5), so they're logically equivalent.

We note that $r$ 's not occurring in $\phi(r, \vec{a})$ is crucial: if it did occur the prenex normal form would be different. As a second note: the crucial step is (4)'s implying (5) - that they are equivalent is an added bonus. And as it happens logics that lack prenex normal form theorems, like intuitionistic logic and most quantified relevance logics would still validate that (4) implies (5). [Thanks to David Charles McCarty for discussion here.] 


\subsection{Solodkoff's Response}

Before giving our own response to deRosset, we briefly discuss Tatiana von Solodkoff's (2012) response. As we see it, her response in effect concedes to deRosset the success of his argument. She ends up rejecting:

(Priority) The fundamental facts do not include any distributions of features over ordinary macroscopic concreta, including tables, raindrops, tectonic plates, galaxies, and the like.

Nevertheless, she proposes (as we'll call it) a "successor project" that she thinks can retain the spirit of Priority Theory, even if rejecting the letter. She aims to retain this spirit by drawing a distinction between natural languages and "Ontologese," which is a "metaphysically primitive language" that carves nature at its joints (2012: 395). On her proposal, the Priority Theorist can provide an explanation of why a raindrop is translucent by giving an explanation that has, as one part, a sentence in Ontologese that does not refer to the raindrop and, second, a "bridge principle" that specifies that an object satisfies 'is a translucent raindrop' when (say) some particular mereological simples are arranged in a certain way. ${ }^{13}$ Because the bridge principle can refer to macroscopic objects, this explanation violates (Priority) (2012: 396-7). But because the first sentence in it - the one in Ontologese - does not refer to the macroscopic object, this successor project (supposedly) retains the spirit of Priority Theory.

Solodkoff's successor project has some interesting additional subtleties we can't address here. Nevertheless, there are several reasons we are wary of her proposal as a response to deRosset's argument. First, her response turns on postulating a certain kind of language, Ontologese. But what exactly Ontologese is, or how it works, or how we can ever be sure we've identified parts of it, are all vexing questions. Bringing in this theoretical machinery makes Solodkoff's response hostage to it. Further, it is not clear that utilizing sentences of Ontologese is well-motivated. If the spirit of Priority Theory is retained by having an explanation containing a sentence of Ontologese that does not refer to macroscopic objects along with a bridge principle that does, then why wouldn't it be retained by having an explanation that contains a sentence of English that does refer to macroscopic objects along with the bridge principle that does? Per (Explanation), the latter would not be legitimate by the lights of any Priority Theorist, since it refers to a nonfundamental thing, so why should the former? To claim that the difference is that Ontologese is a "special, metaphysically explanatory" language or is the part of the explanation that is the "distinctive metaphysical part of the explanation" would seem to dubiously resolve the issue by fiat. ${ }^{14}$ At the very least, the proposals we consider below won't turn on issues involving Ontologese.

Second, recall that (Priority) is implied by

(Explanation) The existence and features of the macroscopic concrete objects alleged by common sense abetted by science can be completely explained solely by reference to the existence and properties of other things.

Thus, to give up (Priority) is to give up (Explanation). But once we've given up (Explanation), we think we have abandoned the spirit of Priority Theory. Solodkoff's approach in effect concedes that we cannot explain the existence and features of macroscopic objects without appealing to them as well. But that amounts to rejecting the distinctive feature of the narrow camp that the Priority

\footnotetext{
${ }^{13}$ The sentence in Ontologese might be picked out using English sentences and a special metaphysical operator, see (2012: 398).

${ }^{14}$ Even stranger, this proposal would require that the explanations Priority Theorists give-specifically, the $e x-$ planans-must frequently straddle two languages. We can't think of any other kind of explanation that is essentially cross-linguistic like this.
} 
Theorist was trying to retain, namely, that macroscopic objects don't play an important explanatory role. So Solodkoff's response might provide the foundations for an interesting ontological projectjust not one a Priority Theorist ought to whole-heartedly embrace, absent further argument. ${ }^{15}$

Whether these gripes about Solodkoff's successor project are right, the most salient point is that the main motivation for her successor project is that it responds to deRosset's argument against Priority Theory. But, since we will be arguing that deRosset's argument is not convincing, this should undermine that motivation.

\section{Problems for the Determination Constraint}

In this section, we critically discuss the P2 premise of deRosset's Main Argument, namely, that no adequate explanation violates deRosset's argument. We identify two problems with it. First, deRosset's argument for it - its derivation as an explication of the (LP) - is suspect. Second, it is remarkably similar to a model of explanation that is not plausibly thought of as providing a general constrain on explanation anyway. Nonetheless, we distill from deRosset's criticism using (Det) an abstract structure that is useful for evaluating Priority Theory - a structure we utilize in the subsequent section.

\subsection{Problems with the Derivation of (Det)}

The (LP) applies when there is a proposed explanation at issue, $r$ is $F$ because $\phi(r, \vec{a})$. (LP) employs the construction, ' $r$ is like $r^{*}$ as far as the explanans goes', 'the explanans' referring back to $\phi(r, \vec{a})$. To get a handle on that locution, let's compare some other uses of the phrase: ${ }^{16}$

Guild Wars 2 [a video game] is like the Academy Awards as far as voice acting goes.

Cactus [a recreational drug] is like acid lite as far as stimulation goes.

\# Wisconsin is like Ohio is like Texas as far as grass goes.

For ease of reference, we'll speak of the three slots of the comparison claim: "[slot 1] is like [slot 2] as far as [slot 3] goes." In these claims, the objects referred to in the first two slots are being compared in terms their possessing, perhaps to some degree, some salient feature invoked by the third slot. For instance, cactus is being compared to acid lite in terms of how "stimulated" it is claimed to make its user feel. Though these statements simply assert a likeness between items in slot 1 and slot 2, it is natural to see them as implying an explanation as to why that likeness occurs. For instance, it is not only that Guild Wars 2 is like the Academy Awards as far as voice acting goes; Guild Wars 2 is like the Academy Awards in virtue of the similarity of their voice acting.

\footnotetext{
${ }^{15}$ Actually, we think her project may be even more unappealing that we are letting on. She actually reformulates the positions in a way that is not logically equivalent to deRosset's. In fact, she reformulates the view under discussion so that it is essentially metalinguistic. E.g., instead of explaining why a raindrop is translucent, metaphysicians are to explain why the English sentence 'this raindrop is translucent' is true. Formulating the issue in this way lets her focus on a position that denies the existence of nonfundamental objects (compare her statement of (Sparsity) (2012: 392) with deRosset's (2010: 76)). But some of the most influential Priority Theorists - like Schaffer or Fine - do not deny the existence of nonfundamental objects and are interested in explaining facts about those objects, not the truth of sentences in a natural language (cf. Fine (2001: 16)). In a footnote she recognizes that the position she is interested is actually inconsistent with the position of authors like Schaffer (2012: $392 \mathrm{fn} 3$ ). But she does not draw the conclusion that such authors are unlikely to embrace a project inconsistent with their own.

${ }^{16}$ These were found by an afternoon of googling. There are a number of wrinkles to the semantics of this expression we can't explore, such as what role context-sensitivity plays in filling out the proposed "likeness." Our discussion will not turn on such wrinkles.
} 
A natural generalization descriptive of these cases is that a sentence fitting the form ' $x$ is like $y$ as far as being $F$ goes' is true only if the salient degree which $F$ applies to $x$ is the same or relevantly comparable to the degree to which $F$ applies to $y$. That certainly seems to be what's going on in the recent examples. Now, if this generalization is right, it ought to at least make sense for the invoked feature $F$ to apply to each of the objects being compared. This point is supported by the seeming anomalousness of the following examples, where what is being invoked by the third slot doesn't seem to apply to either of the first two at all:

(7) The number 3 is like the number 11 as far as astronaut ice cream goes.

(8) Seasons 1 and 2 of True Detective are alike as far as being low in cholesterol goes.

(9) Prince is like Beyoncé so far as the earth has one moon goes.

Read normally, each seems either false or inevaluable. "No, the number 3 is not like the number 11 as far as astronaut ice cream goes," seems like an appropriate response to (7), if a response is even merited. ${ }^{17}$

deRosset's main argument for P2 of his argument is that (Det) is a plausible and natural explication of (LP). Now the (LP) tells us that an explanation of $r$ is $F$ is inadequate if there is some $r^{*}$ such that (i) $r^{*}$ is like $r$ so far as the explanans goes but (ii) $r^{*}$ is not like $r$ so far as the explanandum goes. Let us focus on the first 'is like' locution:

(10) $r^{*}$ is like $r$ so far as the explanans goes

where the explanans of $r$ 's being $F$ is $\phi(r, \vec{a})$. As noted above, deRosset's explication of (LP) amounts to explication this 'is like' talk in (10) in terms of partial satisfaction. Thus, on his understanding (10) is true only if the following is as well:

$\left(10^{*}\right) r^{*}$ is like $r$ so far as partially satisfying $\phi$ (with some $\vec{a}$ ) goes.

And sticking with our observation that these statements imply further statements about why a likeness occurs, if $\left(10^{*}\right)$ is true then presumably so is:

$\left(10^{* *}\right) r^{*}$ is like $r$ in virtue of partially satisfying $\phi$ (with some $\vec{a}$ ) goes.

However, we find $\left(10^{*}\right)$, and by extension $\left(10^{* *}\right)$, bizarre. After all, ex hypothesi, $r$ doesn't actually appear in the object language sentence denoted by $\phi(r, \vec{a})$. Consequently, for the same reason $r^{*}$ would not appear in any "substitution" for $r$, that is, it would not appear in any $\phi\left(r^{*}, \vec{b}\right) .{ }^{18}$ Thus, $\left(10^{*}\right)$ is asking us to compare $r$ and $r^{*}$ in terms of partially satisfying a formula in the "null sense." But we cannot make sense of that. It would be like saying:

$\left(9^{*}\right)$ Prince is like Beyoncé so far as partially satisfying 'the earth has one moon' goes.

Worse yet, $\left(10^{* *}\right)$ states that two things are alike in virtue of partially satisfying a closed formula, and that strikes us as even stranger. Who among us would feel comfortable saying:

$\left(9^{* *}\right)$ Prince is like Beyoncé in virtue of partially satisfying 'the earth has one moon.'

\footnotetext{
${ }^{17}$ For uniformity's sake in what follows we'll stick with the inevaluable/anomalous reading of sentences like (7)-(9); the same points can be made on the false reading.

${ }^{18}$ Unless of course $r^{*}$ is among $\vec{a}$ or $\vec{b}$ or occurs elsewhere in $\phi$, but it can easily be chosen so that this isn't the case.
} 
The problem is that constructions like $\left(10^{*}\right)$ and $\left(10^{* *}\right)$, as well as $\left(9^{*}\right)$ and $\left(9^{* *}\right)$, run afoul of our earlier generalization that such statements are true only if what appears in slot 1 and slot 2 can be meaningfully compared in terms of the salient degree to which they possess the feature invoked in slot $3 .^{19}$

To be clear, we are not questioning Tarski's stipulative usage of 'satisfaction'. deRosset is quite right to claim that the following is true: ${ }^{20}$

(11) Both $r$ and $r^{*}$ partially satisfy $\phi$.

After all, both $r$ and $r^{*}$ satisfy that formula in the "null sense" identified above. What we find bizarre is the idea that $r$ and $r^{*}$ are like each other in virtue of partially satisfying a closed sentence in which neither appears! $!^{21}$

Further, it is no accident that deRosset must say things like $\left(10^{*}\right)$. (Det) is meant to be a principle that is both a natural explication of (LP) and a principle that Priority Theory violates. But insofar as Priority Theory violates (Det) it is only because there are cases where two objects, like our raindrop and a tectonic plate, both partially satisfy a closed formula in which no term denoting them occurs. ${ }^{22}$ Thus, deRosset's explication of (LP) - specifically, his explication of (LP)'s phrase "[slot 1] is like [slot 2] as far as [slot 3] goes"-must permit that one thing is like another so far as partially satisfying a closed formula in which no term denoting them occurs.

But as should be obvious, given how bizarre sentences like $\left(10^{*}\right)$ are, (Det) is not a natural or good explication of (LP). For we see no reason for thinking that (LP) would permit cases like (10*). Thus, insofar as deRosset's main argument for (Det) is that it is a natural explication of (LP), that argument is unsuccessful. And if that argument is unsuccessful, his main argument for the second premise of his critique against Priority Theory is undermined.

In response, deRosset might point out that, generally speaking, the explication of a concept may apply (or be well-defined, or fail to be anomalous) in cases where the to-be-explicated concept did not. ${ }^{23}$ So the mere fact that (Det) extends to cases beyond those that (LP) applies to is not itself problematic. As a general point about explication, we're sympathetic. But we find the particular application here to be problematic nevertheless. First, our objection is not just that (Det) applies to cases that (LP) doesn't; rather, it is that it applies to new cases that are problematically anomalous. Though it may not be constitutive of an explication that it remove anomalous cases, at the very

\footnotetext{
${ }^{19}$ deRosset might urge an alternative understanding of (10). Instead of taking 'the explanans' to refer to a closed sentence, it might instead refer to the function that is used to generate the closed sentence. The idea would be that $r$ and $r^{*}$ are then alike in virtue of being arguments that, in conjunction with other arguments, output a true sentence when fed into the function 'the explanans' refers to. As a reading of (10), we find this forced. Since for reasons explained above Priority Theory would need $\phi$ to be a function that didn't take the argument actually mentioned in (10), to wit, $r$ and $r^{*}$. Ignoring that point, this understanding of (10) would still generate bizarre results. For instance, we could use this proposal to make the nonsensical sentence 'Seasons 1 and 2 of True Detective are alike as far as being low in cholesterol goes' sensible, even true, by appealing to the true sentence: Season 1 and 2 of True Detective are alike in so far as, with some other objects, they partially satisfy ' $x$ is low in cholesterol.'

${ }^{20}$ Since $r^{*}$ is not $F$, it follows that $r^{*}$ is a confounding case for the proposed explanation. Thus, we do not deny that given the way deRosset defines 'confounding case' Priority Theory explanations have confounding cases. The issue is whether deRosset has shown that having a confounding case, in this sense, is ever a problem for a proposed explanation and not simply a quirky formal fact, a mere artifact of a tendentious explication.

${ }^{21}$ Perhaps one reason why this point has gone unremarked in discussion of deRosset's paper is that at no point are any actual explanations given of the kind Priority Theory must traffic in. A general schema (Prop) is specified, with pains to taken to ensure the Priority Theorist's explanation fits it. But it is never instantiated. Some toy examples are discussed, but none fitting the form required by Priority Theory.

${ }^{22}$ If either term actually appeared in the closed formula, deRosset's argument for the first premise would fail, for reasons pointed out in fn. 12 .

${ }^{23}$ Carnap touted this as a benefit, even an aim, of explication (Carnap (1950), chapter 1; see also $\S 53$ of Quine (1960)).
} 
least, the usual point of explicating is to remove such problematic cases, not introduce them! Second, this rejoinder is of dubious dialectical force. For the very kinds of anomalous cases that (Det) applies to are exactly those cases he needs in arguing for the first premise of his argument. A committed Priority Theorist might be forgiven for finding it convenient that the formerly anomalous sentenceconstructions are suddenly given truth-values of exactly the sort that would enable deRosset to run his argument. Thus, anyone - Priority Theorist included - is entitled to reject deRosset's application of (LP) to these explanations, thereby undermining his argument.

\subsection{Is (Det) a Plausible General Adequacy Constraint?}

deRosset pitches (Det) as a general constraint on explanation. But there is reason for doubting that is the case. For (Det) bears some similarities to the Deductive Nomological (D-N) model of explanation, and it is not a general constraint on explanations that they fit the D-N model. To see this, a brief discussion of the D-N model is necessary.

According to the deductive-nomological account of explanation (for exposition see, e.g., Hempel (1965, 1966) or Carnap (1966 [1995])), explanations have the following form. An explanation is a deductive argument that has at least one premise being a "law" (which has the form of a universal generalization) as well as other premises being "initial" or "antecedent" conditions (which usually will entail something that satisfies the antecedent of the universal generalization). In the simplest case, the explanation might appeal to one law and one initial condition. In more complex cases, it might appeal to multiple laws and initial conditions. Either way, the guiding idea behind this model is that explanation is akin to a kind of deduction; understanding deduction through first-order logic fills in this guiding idea.

The D-N account of explanation is very simple and elegant. But as a general account of explanation, it is problematic. There are a variety of problems for it as a model for explanations in either ordinary life or scientific theorizing. ${ }^{24}$ Here we'll focus on just two related problems. First, it is not a general constraint on scientific explanations that the explanans entail the explanandum. The explanans may just make the explanandum very probable or even just more probable than it would be otherwise. To use a well-known example, we might explain why a subject has paresis by appealing to the fact that he has latent untreated syphilis, even if not everyone with latent untreated syphilis develops paresis.

Second, it is not a necessary constraint on an explanation that there be a true "law" understood as a universal generalization. Some scientific disciplines produce explanations in terms of rough generalizations that admit of exceptions in a way that universal generalizations do not. Alternatively, an explanation might not appeal to a law at all, but rather explain why a fact or range of facts obtain by stating the causal antecedents of that fact(s) (cf. Cleland (2011)). Thus, the D-N model is a poor one for scientific explanation in general.

Those points apply equally well when we turn from explanations in scientific contexts to ordinary ones. For instance, if Adam has just turned down a particular appealing piece of cake, Katy might explain why he did so by appealing to the fact that he's on a diet and he doesn't want to spoil it. But clearly that explanans does not entail that he turn down the cake. Just as clearly, Katy's explanation is not foiled because the "law" that everyone who is on a diet and does not want to spoil it does not eat cake is false. Some of the explanations of ordinary life do not fully conform to the D-N model.

\footnotetext{
${ }^{24}$ For critical discussion of this simple model, see Achinstein (1985), Scriven (1959), Railton (1978), Ruben (1990), Salmon (1989), and van Fraassen (1980). Skiles (2015: 742) also critizes a stronger modal form of (Det) that deRosset mentions (2010: 91). Of course, Hempel himself came to think that the D-N account was inadequate and proposed a second one that departed from it in some, but not all, ways.
} 
We mention these points, which will be familiar to some, for the following reason. (Det) faces some of the same problems that the D-N account of explanation does. ${ }^{25}$ For (Det) maintains that the explanatory proposal ' $r$ is $F$ because $\phi(r, \vec{a})$ ' is adequate only if for all $x$, if $x$ partially satisfies $\phi$ then $x$ is $F$. But for those of us who were wary of the D-N account partially because of its insistence that an explanation is adequate only if a certain universal generalizations come out true, we should also be wary of (Det). After all, some explanations are not beholden to universal generalizations. ${ }^{26}$ Thus, P2 from the above argument is false. (Det) is not a general constraint on adequate explanations. ${ }^{27}$

\subsection{Moving Forward}

In this section, we've criticized deRosset's argument, focusing exclusively on (Det) and his derivation of it. But there is a general structure of deRosset's argument that we find promising. The general structure utilizes a proposed adequacy constraint on Priority Theory explanations as well as the fact that such explanations mustn't refer to nonfundamental things to derive a contradiction given the exceedingly plausible assumption that certain facts have an explanation. We think that it is this general structure that is promising for articulating a problem for Priority Theory, not the particular instance of that structure deRosset uses involving (Det). Consequently, we will not consider rejoinders to our criticism that either defend (Det) or try to explicate (LP) to some other

\footnotetext{
${ }^{25}$ Of course, they don't have all the same problems. For instance, proponents of the D-N model have to explain the difference between laws and accidentally true generalizations. Conversely, D-N proponents are not shackled to the extra constraint that a specified term mentioned in the explanandum cannot be mentioned in the explanans.

${ }^{26}$ Once we recognize this, we can see why it is tempting to think of the Determination Constraint as a general constraint on explanations. deRosset's examples implicitly contain, in the explanans, universal generalizations, which he then extrapolates from. For instance, he gives the example: "Diamond is hard because each carbon atom in its crystalline structure is bonded to each of its neighbors" (2010: 78). If you had never had a course in chemistry, this explanation would leave you mystified. For you would not have filled in the other assumed part of this explanation: "All substances which have a crystalline structure in which each carbon atom is bonded to its neighbor are hard." So the explanans implicitly appeals to a universal generalization. But once we see that a fuller articulation of this explanation would include this universally quantified statement, we can see why it might be a poor example to extrapolate from.

${ }^{27}$ An anonymous reviewer worries that our counterexamples to (Det) might be targeting a strawman. As we indicated earlier in fn. 11, deRosset might not intend (Det) as a general constraint. And, as the anonymous reviewer reminds us, deRosset gives toy-examples of the kinds of explanations (Det) should apply to and those examples use the locution 'in virtue of'. So one might worry that because some of our examples are causal explanations, they do not apply. We have a variety of worries to this response. First, even if our criticism of (Det) miss their mark, we think the problem we identity below is more pressing, as we argue. Second, we don't think there is a great gap between causal explanations and "in virtue of" explanations. For instance, to our ears, there's nothing wrong with saying 'Why did Adam turned down the offer of cake? In virtue of the fact that he is on a diet.' Indeed, appealing to the locution 'in virtue of' feels very natural in Scriven's syphilis/paresis case because only patients with syphilis develop paresis, even though this is still a causal explanation. (For more principled reasons to worry about separating out causal explanations from other kinds, see Bennett (2017).) Third, even if we restrict ourselves to non-causal explanations, there might still be counterexamples. Consider an example due to Lipton (2004: 31-32). Throw sticks into the air with spin. If you take a "freeze shot" of them, you'll find that many of them are closer to the horizontal axis then the vertical axis. The explanation of this is, crudely put, that there are more ways for them to be near the horizontal axis then the vertical axis. But this does not imply that any freeze shot of them will fail to capture all of the sticks closer to the vertical axis then the horizontal axis. Without developing them too much, there might be other counterexamples as well. For instance, there might be counterexamples from the normative domain (perhaps we can explain the value of this belief by pointing out it is true without maintaining that all true beliefs are valuable) or perhaps even emergent phenomenon. (For emergent phenomenon in the sciences, see (e.g.) Mitchell (2009), in philosophy O'Connor and Wong (2005).) Finally, we'll just note a dialectical point. As a criticism of Priority Theory, (Det) has more force if it is understood as a general constraint. Restricting it to just a certain kind of explanation Priority Theorists are interested in might make it immune from some criticisms involving causal counterexamples, but it also makes it appear more like a poised pawn.
} 
principle that is distinct from (Det) but could do the work deRosset envisaged for (Det). Rather, we simply move forward to articulating what we think is a promising criticism for Priority Theory that utilizes this structure.

\section{A Problem for Developing Priority Theory}

In this section, we set out a new problem for Priority Theory that reveals a serious constraint on how Priority Theory must be developed not only to be plausible but to be consistent. Like deRosset's, we articulate a proposed adequacy constraint on Priority Theoretic explanations - what we call (Consequence Constraint) - and show how given this constraint Priority Theory will entail a contradiction (\$4.1) given a very uncontroversial assumption. We then show how some natural ways of articulating, or developing, Priority Theory end up implying (Consequence Constraint). That's a problem. We note further in $\S 4.6$ that (Consequence Constraint) is even stronger than it needs to be. Even articulations of Priority Theory that eschew (Consequence Constraint) may still license the contradiction-entailing reasoning and so fall prey to this problem if they embrace a much weaker principle we call (Minimal).

\subsection{Deriving a Contradiction}

In this section we introduce a constraint on Priority Theoretic explanations. ${ }^{28}$ To exhibit how the contradiction follows, we simply assume this it is a constraint here. Then in subsequent subsections we'll identify various reasons Priority Theorists might be inclined towards accepting it.

(Consequence Constraint) If something $r$ is nonfundamental and $r$ is $F$, where $F$ is non-total, then there is a set of true sentences $\Gamma$ where no sentence in $\Gamma$ mentions $r$ and $\Gamma \mid=r$ is $F$.

By the symbol $\models$ we have in mind a consequence relation that is an admissible extension of the usual consequence relation of classical, first-order logic. ${ }^{29}$ As we have seen, by the Priority Theoretic account of fundamentality, if $r$ is nonfundamental then there must exist some explanation of $r$ is $F$ that makes no mention of $r$ in its explanans. So it is tempting to read the $\Gamma$ in $(\mathrm{CC})$ as just that explanans of the relevant explanation. As we will see, however, (CC) needn't be understood so narrowly. If $r$ is $F$ it is enough (for the purposes of deriving a contradiction) that there is some set of true sentences that $\models$ implies it.

Here then is how (CC) would get Priority Theory into trouble. We assume Priority Theory isn't even worth considering if it isn't consistent with there being a nonfundamental entity that has a non-total property. So let $r$ be nonfundamental and $F$ be non-total. It follows, by (CC), that

(12) There is a set of true sentences $\Gamma$ and $r$ doesn't occur anywhere in $\Gamma$ and $\Gamma \models r$ is $F$.

But (12) implies:

(13) $\Gamma \models(\forall x)(x$ is $F)$.

\footnotetext{
${ }^{28}$ Priority Theory is concerned with a certain kind of metaphysical explanation. In this section, any explanation mentioned is to be understood as a "Priority Theoretic" explanation, whatever that happens to be.

${ }^{29} \mathrm{An}$ admissible extension of a consequence relation is a consequence relation that preserves all theorems of the original. We interpret $=$ in this way primarily for reasons of familiarity and expedience. Strictly, the present argument could be given with any (CC)-like condition stated in terms of consequence relation that it is truth-preserving, in that true premise sets should only have as $\models$ consequences true conclusions, and "normative," in that it should model certain logically impeccable forms of reasoning. (The impeccable forms of reasoning at issue will always be deductive or nonampliative. Thanks to an anonymous referee.) The argument in $\S 4.5$ is framed in terms of these two more abstract conditions.
} 
That (12) implies (13) follows from a metatheorem familiar from mathematical logic called the Generalization on Constants:

(GoC) If $\Gamma \models \phi(r)$ and $r$ doesn't occur anywhere in $\Gamma$ then $\Gamma \models(\forall x) \phi(x) .{ }^{30}$

Since by assumption $\Gamma$ is true and $\models$ is truth-preserving, it follows from (13) that $(\forall x)(x$ is $F)$. But that contradicts our assumption that $F$ was non-total. Thus, any version of Priority Theory on which (CC) comes out true will imply a contradiction.

A crucial step in the reasoning is the Generalization on Constants, (GoC). We claim that all things equal, (GoC) ought to hold for any consequence relation that might be applied in contexts like the present one. (GoC) is a formal means of capturing an important kind of reasoning that any consequence relation should model, that of deriving a universal generalization from an arbitrary case. As an example, suppose one wanted to prove some general condition $\phi(x)$ holds of some class, say, that any prime number greater than 2 is odd. Then one could begin by introducing a constant, $n$, to stand for some arbitrary prime number greater than 2. Upon showing that the arbitrary $n$ is odd it would follow that any prime numbers greater than 2 are odd. (GoC) is a way of modeling this reasoning. The constant $n$ 's being arbitrary is modeled by the fact that $n$ is mentioned nowhere in the premises $(\Gamma)$. Since none of our premises mention $n$, they could not make any substantive assumptions about it, and in that sense it is arbitrary. Presumably we want a consequence relation to capture this eminently impeccable form of reasoning, and so we assume that any logic that Priority Theorists are drawn to will contain a $=$ relation that validates $(\mathrm{GoC}) .{ }^{31}$

This line of reasoning is similar deRosset's reasoning in that both derivations use an alleged constraint on adequate explanations to derive a contradiction. But there are some differences worth noting. First, we do not assume that all explanations can be regimented in line with (Prop). Since we do not need to regiment in (Prop), we do not need to make use of deRosset's arguably contentious notation. Second, deRosset's derivation was premised on (Det) as a constraint, ours was premised on the Consequence Constraint, (CC). As it happens, the line of reasoning we use actually subsumes deRosset's: (Det) implies (CC) but not conversely. Here is a simple reason (CC) does not imply (Det). (Det) makes claims about non-Priority Theory explanations, but (CC) does not. Thus, (CC) does not imply (Det). But (Det) does imply (CC). To see this, recall the formal reasoning presenting in section 2.3. There we showed how the result of applying (Det) to a the usual explanans proposed Priority Theory explanans in conjunction with that explanans will imply the universal generalization of the relevant explanandum. To use the example of $r$ is $F$, we showed that:

$$
\phi(r, \vec{a}),(\forall x, \vec{y})[\phi(x, \vec{y} \rightarrow F x] \models(\forall x) F x
$$

where none of the elements on the left mention $r$ for the standard reasons. But clearly if a set of sentences implies the universal generalization of an explanandum, it will also imply the explanandum. Thus, if (Det) is true, then if there is an adequate Priority Theory explanation of some fact,

\footnotetext{
${ }^{30}$ Cf. Enderton (2001: 123 - 124), Hinman (2005: 176).

${ }^{31}$ We say that all things equal (GoC) ought to hold for a consequence relation because it is merely sufficient for modeling the desired reasoning, if rather naturally so, but it may not be necessary. One way around the challenge we are posing in this section is to show how to license this reasoning without (GoC). But exploring other ways of modeling this reasoning would take us too far afield - we're primarily interested in posing the challenge.

We also note that $(\mathrm{GoC})$ is not the only metatheorem that can be slotted into the crucial step in the derivation of the contradiction. The derivation could also be run, with some extra effort, with The Craig Interpolation Theorem in place of (GoC) (cf. Fefferman (2008)). Alternatively, if $=$ were a relevant consequence relation then it will have as a metatheorem a variable-sharing property: that at least one sentence in one of the premises $(\Gamma)$ occur in the conclusion $(\phi)$ (Mares (2019)). With a bit of extra effort this metatheorem could be slotted into the derivation as well. Again, our interest is in tracing the quickest logical path to the challenge, and to exhibit some philosophical commitments that would lead to it.
} 
then there is also some set of sentences that do no mention the objects in that fact but nonetheless imply that the fact obtains. But that is just what (CC) says. Thus, (Det) implies (CC).

Finally, even if deRosset's derivation of a contradiction and ours are similar, there is a difference to keep in mind: their starting point. We've already argued that deRosset's starting point - (Det) is one Priority Theorists are on good grounds to reject. But, as we'll show in subsequent sections, our starting point- $(\mathrm{CC})$ - is one that several self-conscious Priority Theorists will be drawn to. This makes (CC) and by extension our problem a more promising object of study.

\subsection{The Challenge}

The basic problem for Priority Theory could be understood as a challenge: a challenge to give principled reasons for thinking their theory could be developed in such a way as to not only avoid (CC) as a general constraint but also every instance of (CC) that would trigger the derivation of the contradiction given the uncontroversial assumption. After all, if Priority Theory implies even one instance of (CC), such a derivation will be possible.

What makes this a challenge is that there are, in principle, several ways of implying an instance of (CC). For all (CC) says is that there is a set of truths that implies some explanandum which nowhere mentions the objects in the explanandum. It says nothing else about the sentences that make up this set or their explanatory status vis-à-vis the explanandum. Thus, one way for Priority Theory to imply an instance of (CC) is for its explanans to imply the explanandum; another is for some proposed adequacy constraint on Priority Theoretic explanations to imply the explanandum without mentioning the relevant objects; a third way is for the conjunction of an explanans along with such an adequacy constraint on that explanans to imply the explanandum without mentioning the relevant objects. Any of these ways would spell trouble for Priority Theory. ${ }^{32}$

As we see it, it is this third way of implying an instance of (CC) that is most pressing. For Priority Theory is ambitious in that it seeks to provide an alternative way of doing ontology that places questions of explanatory priority at the center instead of questions of what exists. To develop Priority Theory into an approach that is as well-understood as the Quinean approach to ontology, Priority Theorists should tell us more about the kinds of explanations they see as central to ontology. And a natural way to do that is to tell us more about what those explanations must be like; that is, to tell us what the adequacy constraints on their explanations are. But the more adequacy constraints they propose, the more they run the risk that those adequacy constraints, when applied and then combined with an explanans, will actually imply the relevant explanandum without mentioning the objects in the explanandum.

In what follows, we'll explore this challenge by looking through a couple of possible adequacy constraints Priority Theorists might be drawn to. We'll show how they actually end up implying (CC). We'll start with some simple cases before moving to more complex ones. We focus on several ways Priority Theory might imply (CC) to illustrate the depth of the problem, that is, to make good on our claim that it can be challenging to develop Priority Theory in a way that actually avoids (CC). ${ }^{33}$

\footnotetext{
${ }^{32}$ This provides another reason for thinking (Det) and (CC) differ. (Det) implies that what entails the explanandum will also contain the explanans. (CC) does not imply that.

${ }^{33}$ To be sure, more could be said on each constraint in an attempt to foist it upon the Priority Theorist. But we find that style of argumentation more dialectically fraught. Besides, focusing on just one constraint would miss the bigger picture of the difficulty of developing Priority Theory.
} 


\subsection{The God Metaphor}

Consider a common metaphor employed by Priority Theorists, that God in creating the world need only to speak of - or think of, or act on - the fundamental things. In making the world it would have been unnecessary for God to pay separate heed to the nonfundamental. Some representative quotes:

"There is a second assumption [...] that I think we ought to make-what I call "purity": fundamental truths involve only fundamental notions. When god was creating the world, she was not required to think in terms of nonfundamental notions like city, smile, or candy." (Sider (2011: 125, cf. 171))

"[T]he neo-Aristotelian will begin from a heirarchical view of reality ordered by priority in nature. The primary entities form the sparse structure of being, while the grounding relations generate an abundant superstructure of posterior entities. The primary is (as it were) all God would need to create." (Schaffer (2009: 351, our emphasis)) ${ }^{34}$

In this metaphor, the nonfundamental facts arise automatically out of, are secured by, are grounded in, or come for free given the fundamental facts. Conversely, the fundamental facts are said to determine the nonfundamental facts (cf. Audi (2012), Wilsch (2016)).

A natural way of cashing out this idea - of what follows ineluctably from God's creations - is as a consequence relation: that being automatically secured (or whatever) is a consequence relation on facts (say). ${ }^{35}$ For, assuming that multiple facts may jointly determine another fact, then the relation of determination at issue will at least have the form of a consequence relation:

\begin{tabular}{lccc}
\hline consequence: & sequence of premises & implies & single conclusion \\
determination: & sequence of facts & determines & single fact \\
\hline
\end{tabular}

And it makes sense to say that insofar as some fundamental facts (jointly) determine a nonfundamental fact, they will suffice for, we could even say entail, the nonfundamental fact's existence. This all suggests that Priority Theorists drawn to this God metaphor should cash that metaphor out by endorsing the following principle:

(Entailment) $\Delta$ provides an adequate explanation of $\phi$ only if $\Delta \mid=\phi$.

(Entailment) can be seen as an adequacy constraint on Priority Theory explanations insofar as it sets necessary conditions on those explanations.

However, by definition, if $\Delta$ provides a Priority Theory explanations of $\phi$, then whatever objects are mentioned in $\phi$ are not mentioned in $\Delta$, and $\Delta$ should be true. But given that $\Delta \mid=\phi,(\mathrm{CC})$ follows. Thus, if (Entailment) is true, so is (CC). And if (CC) is true, then Priority Theory is incoherent.

To be sure, a Priority Theorist might reply that these ways of introducing their distinction are only metaphors, and either cash it out some other way or see it as a Wittgensteinian ladder to be kicked away upon understanding the view and never to be cashed out. But the metaphors are scratching a certain itch about how the fundamental relates to the non-fundamental. If that itch is that the fundamental entails the non-fundamental, then Priority Theory is incoherent.

\footnotetext{
${ }^{34}$ Of course, this metaphor is not new; Kim (1979: 40-41) used it to make a similar point.

${ }^{35} \mathrm{It}$ is not for nothing that Bolzano appears to be have thought of his notion of grounding as an explanatory consequence relation (Roski \& Rumberg (2016, p. 473), Roski (2017, Chapter 2)). And then there is Wittgenstein from the Tractatus:

"If a god creates a world in which certain propositions are true, then by that very act he also creates a world in which all the propositions that follow from them come true." (Wittgenstein (1922) §5.123.)

${ }^{36}$ When convenient we will work with sets of premises instead of sequences.
} 


\subsection{Metaphysical D-N \& Laws of Metaphysics}

Another way of developing Priority Theory is basing it off the D-N model of explanation. On this view, while the $\mathrm{D}-\mathrm{N}$ model may not be a good model for explanation most generally, it is a good model for the kind of explanations that Priority Theorists are interested in. On such a view, there are "metaphysical" laws. The particularities of the laws might depend upon different philosophical views. Examples of potential laws might be things like: whenever certain neural networks fire in particular ways, then the agent who has those neural networks, say, is thinking of ice cream; or if certain atoms are arranged in a structure $S$, then they compose an object that is fragile. In general, these metaphysical laws will be general truths linking more fundamental things to less fundamental things. Indeed, this way of developing Priority Theory could even be understood as filling in the idea that the fundamental "determines" the non-fundamental - it determines it in accordance with metaphysical laws.

The most straightforward way of developing a metaphysical D-N model would be to hold that Priority Theory explanations contain in their explanans a metaphysical law or set of laws that, with the help of other truths, entail the explanandum. Thus the adequacy constraint:

(Metaphysical D-N) $\Delta$ is an explanation of $\varphi$ only if among $\Delta$ is a set of laws $\Lambda$ and $\Delta(=\Delta \cup \Lambda)$ $=\varphi$.

Once again, (Metaphysical D-N) can be seen as a proposed adequacy constraint on Priority Theory explanations, insofar as it sets necessary conditions for them. However, (Metaphysical D-N) straightforwardly implies (CC). If $\Delta \cup \Lambda$ explains $\phi$, then $\Delta \cup \Lambda$ cannot mention the objects in $\phi$. But it also implies $\phi$. Thus, there is a set that implies that relevant explanandum without mentioning the objects in it, which is what (CC) says. Thus, any version of Priority Theory that is developed to imply (Metaphysical D-N) is incoherent.

There's a more sophisticated way of developing a version of Priority Theory using a D-N model. Instead of having metaphysical laws be a part of the explanans of Priority Theoretic explanations, they can be understood as "side conditions" for the adequacy of a Priority Theory explanation. ${ }^{37}$ The idea would then be that metaphysical laws are not part of explanations but rather "back them." Of course, to be a proper Deductive-Nomological model of explanation, it should be the case that the explanans when conjoined with the laws entails the explanandum. This gives us the following the adequacy constraint:

(Modified Metaphysical D-N) $\Delta$ is an explanation of $\phi$ only if there is a law or set of laws $\Lambda$ such that $\Delta \cup \Lambda \models \phi$.

On this modified view, it is not required that the explanans $\Delta$ entail the explanandum, which is enough to undercut the argument of the previous section against (Metaphysical D-N).

Nevertheless, this more nuanced approach does not avoid the problem. What matters to (CC) is simply that there is some set $\Gamma$ that does not mention (e.g.) $r$ but also implies that $r$ is $F$. As we noted, it does not matter if $\Gamma$ is itself the explanans for $r$ is $F$. On (Modified Metaphysical D-N) it will be the case that there is a $\Gamma$ such that $\Gamma \models \phi$, where $\Gamma=\Delta \cup \Lambda$. For $\Lambda$ is a collection of laws that, while not part of the explanation of $\phi$, do imply $\phi$ given $\Delta$. Thus, treating the law or collections of laws as a side condition instead of part of an explanans does not resolve the problem.

To be sure, the reasoning leading to the contradiction is only triggered if in the relevant $\Delta \cup \Lambda$ the law(s) $\Lambda$ themselves contain no reference to $r$. But that's a plausible assumption to make. It is

\footnotetext{
${ }^{37}$ Wilsch (2016) seems to defend a version of this view. Similarly, Skow (2016: 110-1) suggests laws of nature play a role in explaining why (e.g.) the microscopic structure of a vase explains its fragility, without those laws being part of the reason, i.e. the explanans, for the vase's being fragile.
} 
hard to understand laws as backing some fundamental explanation of an atomic fact if instead we insisted that the laws themselves always mention the individual in the atomic fact, as we would have to to avoid licensing any instance of (CC). There is an enormous number of atomic facts involving non-total properties - a particular rain drop is translucent, this coffee mug is fragile, Chicago has a lively downtown, etc. It would be absurd to hold that for each such fact there is a metaphysical law that mentions each of these particular things. ${ }^{38}$

One lesson from of the challenge, as it applies the metaphysical D-N model of Priority Theory, is that proponents of that model need to say about what plays the role of the metaphysical laws to show that their account doesn't end up furnishing the premises of the contradiction-entailing reasoning we set out in $\S 4.1$. We think this point applies even to the account of metaphysical laws presented in Glazier (2016), one of the most sophisticated accounts on offer. ${ }^{39}$ Glazier characterizes metaphysical laws as facts which play the necessary role of connecting explanans and explanandum in a metaphysical explanation. He presents his account by reference to the toy explanation, 'The barn is red because the barn is crimson.' (p. 23) In this case the connecting fact, which he calls 'the Connection', “... states the general metaphysical-explanatory connection that holds between an arbitrary thing's being crimson and its being red." Glazier (2016:26; our emphasis) Now, since the connection is between arbitrary things, it is tempting to read the latter as a universal generalization: all crimson barns are red barns. But the perils of taking metaphysical laws to be universal generalizations should be well-established by this point. Happily, Glazier argues separately that such Connection facts are not universal generalizations; they have a different logical form. ${ }^{40}$ The trouble is they still might be logically related to relevant the generalizations:

It may be that [the Connection] is materially equivalent to some such universal generalization. But even if this is so, there is reason to think the two are distinct facts. (Our emphasis, p. 24)

Even if these so-called Connections differ from universal generalizations as facts, provided they imply the relevant universal generalizations, Glazier's account is subject to the challenge just as much as less sophisticated accounts. ${ }^{41}$ The challenge facing the proponent of metaphysical laws is how to secure the desired "generality" of their envisaged laws, without also implying propositions that will get them into trouble.

\subsection{Grounding and Consequences}

A final example. Many proponents of Priority Theory are grounding theorists who see the fundamental as grounding the nonfundamental. ${ }^{42}$ We'll sketch a reason for thinking that even this way of developing Priority Theory may license something like (CC) from which the derivation of a contradiction can be made. The details in this case are more complex. But working through this

\footnotetext{
${ }^{38}$ This point is consistent with their being laws about rains drops, coffee mugs, and downtowns in general. (Though we might have worries about there being such laws.) For what its worth, in Fisher (unpublished), we discuss this position at a greater length. We argue that this position actually licenses a regress - one more general than that identified by Bennett (2011) and discussed in Sider (Forthcoming) and deRosset (2013).

${ }^{39}$ We should add that a lot of the language in that article is tentative and exploratory, so we don't want to insist too much here. Plus there is much in that paper, including the rich discussion in $\S 1.6$, that we can't discuss here, just for reasons of space. Still, nothing in the later sections directly addresses the challenge we are posing here.

${ }^{40}$ cf. (2016: 23ff.)

${ }^{41}$ In the inset quote, the universal generalization Glazier actually cites is 'For all $x$,if $x$ is crimson, then that $x$ is crimson makes it the case that $x$ is red.' (p. 23) But since $\psi$ makes it the case that $\phi$ implies the material conditional, $\psi \rightarrow \phi$, this more complicated generalization logically implies the simpler universal generalization given above.

${ }^{42}$ See essays in, e.g., Correia \& Schnieder (2012), Chalmers, Manley, Wasserman (2009), or the citations in Bliss \& Trogdon (2014).
} 
more complex case is important because this version of Priority Theory is currently quite prevalent. Moreover, this case indicates once again the variety of ways that Priority Theory might be problematic and thus increases the challenge for them to articulate and defend a version which avoids the problem we identify.

Let us assume that Priority explanations are licensed by grounding relations. ${ }^{43} \mathrm{We}$ write grounding relations canonically as $\left[\psi_{1}\right], \ldots,\left[\psi_{n}\right]>[\phi]$, where $[\psi]$ is shorthand for 'the fact that $\psi$ '. Thus the adequacy constraint:

(Grounding Explanation) If $\phi$ because $\psi_{1}, \ldots, \psi_{n}$ is an adequate explanation, then $\left[\psi_{1}\right], \ldots,\left[\psi_{n}\right]$ ground $[\phi]$ (i.e., $\left[\psi_{1}\right], \ldots,\left[\psi_{n}\right]>[\phi]$ ).

Unlike the previous three constraints, (Grounding Explanation) makes no reference to a consequence relation like $=$. At first blush, this may suggest that it will avoid implying anything like (CC). We show this is mistaken.

First, let us say a consequence relation $\Rightarrow$ extends the grounds relation $>$ if: $\psi \Rightarrow \phi$ provided that $[\psi]>[\phi] .{ }^{44}$ Now consequence relations and grounding relations are commonly held to have different formal properties. A consequence relation is reflexive, monotonic, and transitive. Grounding is irreflexive, nonmonotic, and (arguably) transitive. ${ }^{45}$ However, the difference in formal features is irrelevant to our argument. We are not claiming that grounding is a consequence relation, only that there is a consequence relation $\Rightarrow$ that extends it. Here is one such extension.

We define a derivability relation similar to the usual notion of derivability from proof theory, but instead of steps in the derivation being licensed by inference rules of some calculus they are licensed by grounding relations that obtain. As always a derivation starts with a set of premises, $\Gamma$. A sequence of sentences counts as a derivation if it can be constructed by repeated application of the derivation rules. There are two derivation rules. First, a member of the set of premises may be written on a line at any point. Second, one may write $\phi$ on a line if $\left[\psi_{1}\right], \ldots,\left[\psi_{n}\right]>[\phi]$ and each of $\psi_{1}, \ldots, \psi_{n}$ occur in earlier lines in the sequence. (Intuitively: if each of what are the grounds of $[\phi]$ are either assumed or derived then one can take $[\phi]$ to be the case as well. After all, the grounds of $[\phi]$ are supposed to "give rise" to $[\phi]$, so they should at least be jointly sufficient for $[\phi]$.) We write $\Gamma \Rightarrow \phi$ if there exists a derivation in this sense which utilizes the members of $\Gamma$ as premises. ${ }^{46}$

Given the way $\Rightarrow$ was constructed, $\Rightarrow$ extends $>$. That is:

(Extends) If $\left[\psi_{1}\right], \ldots,\left[\psi_{n}\right]>[\phi]$, then $\psi_{1}, \ldots, \psi_{n} \Rightarrow \phi$.

And so (Grounding Explanation) and (Extends) together imply a further adequacy constraint on grounding explanations:

(Extended Grounding Explanation) If $\phi$ because $\psi_{1}, \ldots, \psi_{n}$ is an adequate explanation, then $\psi_{1}, \ldots, \psi_{n} \Rightarrow \phi$.

Syntactically, (EGE) looks like the Consequence Constraint (CC) that we used in $\S 4.1$ to derive a contradiction. In fact, they're the same principle just with $\Rightarrow$ swapped for $\models$. Now the derivation of a contradiction using (CC) turned on two features of $\models$. First, that $\models$ is truth-preserving. Second, that $=$ models what we called generalizing from an arbitrary case. $\mid=$ models that form of reasoning using (GoC). A sufficient condition for (EGE) to derive a contradiction, and thus a problem for this version of Priority Theory, is if $\Rightarrow$ has both of these features. We argue briefly that it does.

\footnotetext{
${ }^{43}$ Fine (2012), deRosset (2013), Trogdon (forthcoming)'.

${ }^{44}$ Here we mean a formal consequence relation in the sense first set out by Tarski (2002).

${ }^{45}$ Schaffer (2009) argues against transitivity.

${ }^{46}$ Correia (2015) also compares grounding to consequence relations.
} 
First, $\Rightarrow$ is a truth-preserving consequence relation. It is a (formal) consequence relation because it is reflexive, monotonic, and transitive. It is truth-preserving because of the way it was defined in terms of $>$ and the fact that $>$ is truth-preserving. ${ }^{47}$

Second, $\Rightarrow$ models the form of reasoning from an arbitrary case. Our case here is defeasible in nature. There are many logically impeccable forms of inference $\models$ models that $\Rightarrow$ models as well. This gives us reason for thinking $\Rightarrow$ will also model the form of inference of reasoning from an arbitrary case that $\models$ models. Our case here is defeasible because we lack a proof that $\Rightarrow$ will model all of the patterns of inference that $\models$ does. But the case does firmly place the burden of proof on those defenders of Priority Theory to explain how it is that $\Rightarrow$ would fail to model a certain kind of inference while modeling so many others.

In speaking of modeling impeccable inferences, we do not having anything fancy in mind. An inference has two parts, a set of premises $\Gamma$ and a conclusion $\phi$. A consequence relation, $\models$ say, models an inference directly if $\Gamma \models \phi$. For instance, it would be impeccable to infer $\psi$ from premises $\phi \rightarrow \psi$ and $\phi . \mid=$ models that inference because $\phi, \phi \rightarrow \psi \models \psi$. But the relation may also model an inference indirectly, for which it suffices that $\Gamma^{*} \models \phi$ for some set $\Gamma^{*}$ logically equivalent to the set of premises. For example, it is uncontroversial that a truth-functionally complete logical language with only disjunction and negation may nevertheless model an impeccable inference from a conjunction by modeling the conjunctive sentences as logically equivalent disjunctions.

For one, $\Rightarrow$ models directly many of the impeccable inferences that $\models$ models. If two things are true, then clearly it is logically impeccable to infer their conjunction. And if something is true, then it is logically impeccable to infer a disjunction where it is one of the disjuncts. $=$ captures these rules of inference using well-known introduction rules for conjunction and disjunction. And many grounding theorists adopt straightforwardly analogous rules for the introduction of $>$, e.g.:

$(\wedge$-in) $\psi, \phi>\psi \wedge \phi$

$\left(\vee\right.$-in) $\psi>\psi \vee \phi ; \phi>\psi \vee \phi^{48}$

Given the way that $\Rightarrow$ extends $>$, it follows that it will model these impeccable inferences that $\models$ does.

On the other hand, there are a number of impeccable inferences that $\Rightarrow$ models but more indirectly. Here is one example. It is an impeccable inference to infer from a conjunction one of its conjuncts. $\models$ models this directly using well-known rules of conjunction elimination: $\phi \wedge \psi \models \phi$. But it is not generally the case that $\phi \wedge \psi>\phi$. Thus, given the way $\Rightarrow$ is defined, it will not generally be the case that $\phi \wedge \psi \Rightarrow \phi$. Nonetheless, $\Rightarrow$ models the impeccable inference indirectly, since $\phi, \psi \Rightarrow \phi{ }^{49}$ Another example. If $\phi$ is false and either $\phi$ or $\psi$ is true, it is impeccable to infer that $\psi$ is true. $\models$ models this disjunction elimination reasoning as: $\neg \phi, \phi \vee \psi \models \psi$. It may not in general be the case that $\neg \phi, \phi \vee \psi \Rightarrow \psi$. But $\neg \phi, \phi \vee \psi$ is logically equivalent to $\neg \phi, \psi$ and it will in general be the case that $\neg \phi, \psi \Rightarrow \psi{ }^{50}$ In this way, $\Rightarrow$ models the impeccable inference, albeit indirectly.

\footnotetext{
${ }^{47}$ The definition of $\Rightarrow$ above is just an intuitive way of defining what is the reflexive closure of the monontonic closure of the transitive closure of the relation, $>$. (The transitive closure of a relation is the smallest extension which is transitive. Likewise for the other closures.) It is easy to show that the property of being truth-preserving is itself preserved under each of these closure operations.

${ }^{48}$ See Fine (2012)

${ }^{49}$ Justification: $\Rightarrow$ is reflexive, so $\phi \Rightarrow \phi$; it is also monotonic, so $\phi, \psi \Rightarrow \phi$.

${ }^{50}$ The justification here is essentially the same as the last one (conjunction elimination): it suffices for the consequence relation $\Rightarrow$ to model an impeccable form of reasoning that $\models$ does if $\models$ models that reasoning with $\Gamma \mid=\phi$ and there exists a set of sentences $\Gamma^{*}$ straightforwardly logically equivalent to $\Gamma$ such that $\Gamma^{*} \Rightarrow \phi$. To model a form of reasoning one may "pivot" on a logically equivalent set of premises. To bring it back to the God metaphor: presumably, God in making something the case thereby also makes everything logically equivalent to it the case.
} 
These considerations provide defeasible reason for thinking that $\Rightarrow$ models many if not all of the impeccable inferences that $\models$ does. But, recall, $\models$ models generalizing from arbitrary cases with (GoC). Thus, we have reason for thinking that $\Rightarrow$ will likewise validate $(\mathrm{GoC})$ so as to model generalizing from arbitrary cases. However, by well-rehearsed reasoning, if $\Rightarrow$ is a truth-preserving consequence relation that validates $(\mathrm{GoC})$, then (Extended Grounding Explanation) - and thus (Grounding Explanation) - will imply a contradiction. So even versions of Priority Theory that understand that view in terms of grounding are not necessarily in the clear.

It bears repeating that we are not claiming that grounding relations are themselves consequence relations and our argument does not require that claim. What this argument needs is that there is a truth-preserving consequence relation that extends grounding claims while also validating (GoC). To be sure, there are important differences, including important formal differences, between $>$ and $\Rightarrow$. But, at this point, the burden is on the grounding theorist to explain why such differences would show the above argument mistaken. And doing so would amount to what we seek: giving principled reasons for why this version of Priority Theory is immune from the problem we articulate.

\subsection{The Robustness of the Problem}

We've briefly shown how several distinct ways of thinking about Priority Theory leads to a contradiction given an uncontroversial assumption. That there are several ways indicates the robustness of the problem. But each of the ways we've identified contains a bit of overkill. If (CC) is true, then each Priority Theory explanation of a non-total fact will imply a contradiction. But clearly for these ways of thinking about Priority Theory to be problematic it is not necessary that they frequently imply a contradiction-just one will do.

There is a more minimal way in which Priority Theory might derive a contradiction. Suppose the following is true:

(Minimal) There is some fact, say that $s$ is $G$, with $G$ non-total, and set $\Gamma$ that doesn't mention $s$, where $\Gamma \models s$ is $G$.

(Minimal) derives the contradiction identified above - i.e., $\Gamma$ will derive that everything is $G$, despite ex hypothesi not everything being $G$. To be consistent, Priority Theory must reject (Minimal) and anything that would imply it. Thus, merely to be consistent, Priority Theory must maintain that no explanation of a non-total fact actually implies that fact.

Further, Priority Theorists might be drawn to various general adequacy constraints on their explanations, constraints like (Det), (CC), (Entailment), (Metaphysical D-N), (GC), or others yet. To avoid implying (Minimal), they must maintain that any instance of a proposed constraint, when applied to an explanation of a non-total fact, either refers to the objects mentioned in the non-total fact, or does not imply the explanandum when combined with the explanans.

Those constraints are all general constraints applying to any Priority Theory explanation. But Priority Theorists might also be drawn to specific constraints to specific domains of facts. For instance, there might be distinctive constraints for explanations of logical and mathematical facts, or normative and evaluative facts, or scientific facts, or modal facts, or supernatural facts etc. And, again, for any Priority Theory explanation of a particular non-total fact in any domain, it must be the case that all of the constraints, when applied to that explanation, either (i) refers to the objects mentions in the non-toal fact or (ii) does not imply the explanandum when combined with the explanans.

To use a metaphor, merely to be consistent, Priority Theory must erect a "wall" prohibiting implication between the non-total facts and whatever apparatus they bring to bear on explaining or 
understanding those non-total facts. ${ }^{51}$ This wall must itself be total — if it breaks down at a single place, for a single non-total fact, then Priority Theory will be inconsistent. ${ }^{52}$

This point has sometimes been lost on readers of this paper. Some have pointed out that philosophers have not always been concerned with logical consequence but "metaphysical" necessitating, but since the later is a more restrictive than former, such philosophers cannot be bothered by our problem. It has been suggested to us that even among those concerned with metaphysical consequence, they might reject it as a general constraint (e.g., Skiles (2015)). Others yet have suggested that metaphysical laws might be vague (see Wasserman (2017)) and thus neither equivalent to or implying universal generalizations. But they at best show that (CC) is rejected by these philosophers; they do not in and of themselves give any reason for rejecting (Minimal). But (Minimal) is all that is needed to generate a contradiction. To use a simplifying analogy, in previous sections, we've argued that several views imply that all As are Bs. In response, some have pointed out that not everyone would agree that all As are Bs. However, the problem is that even if there is a single A that is a $\mathrm{B}$, a contradiction can be derived. But clearly pointing out that, on some view, not all As are Bs does not show that, on that view, no A is a B.

While developing the position, there might be several ways that Priority Theory implies (Minimal) without implying (CC). For instance, perhaps there is a distinctive constraint on Priority Theory explanations for some domain of facts that, when applied to explanations in that domain, implies (Minimal). Or perhaps facts of a certain structural or logical form that cause problems. (E.g. General constraints on Priority Theory, when applied to explanation of these facts, implies (Minimal).) Or perhaps there are certain proposed constraints that are defined only for certain situations that are simply undefined for others; yet those constraints, when applied to situations where they are defined, imply (Minimal). To be consistent, proponents of Priority Theory must eschew all such situations.

And of course Priority Theory aspires to be more than just tenable. It aspires to be plausible, so plausible as to justify a re-orientation of our ontological theorizing. To have such a status we need some principled reasons for why it should be developed in such a way as to avoid (Minimal) and anything that would imply it. In fact, we should seek a principle or cluster of principles that ensures that (Minimal) is false. Anything less than that will leave us in doubt that there is some contradiction derivable from Priority Theory's explanations - a doubt that is surely not groundless given not only the enormous range of non-total facts there are but the number of ways that developing Priority Theory can derive a contradiction.

\subsection{Summing Up}

Thus, there is a substantive problem for developing Priority Theory that several natural ways of developing Priority Theory do not avoid. To be sure, this problem does not demonstrate that Priority Theory is incoherent. Priority Theory is akin to a research program, and it is very difficult to prove any research program incoherent. But it does put the burden on proponents of Priority Theory to show that their research program is not, in Lakatos' charming phrase, a degenerating research program. ${ }^{53}$

\footnotetext{
${ }^{51}$ Perhaps along the lines of Russell \& Restall (2010)'s barriers to implication.

${ }^{52}$ This is why the results of $\S 3.2$ do not help Priority Theorists. Even if it need not be the case that explanans must entail explanandums in general, which we find plausible, they must never entail in this class of cases for Priority Theory to be consistent.

${ }^{53}$ Thanks to Tim Leisz, David Charles McCarty, Nick Montgomery, Tim OConnor, Harrison Waldo, Phil Woodward, audiences at the Society for Exact Philosophy, and two anonymous reviewers.
} 


\section{Conclusion}

Priority theory is an important and interesting view in contemporary metaphysics. With more and more philosophers turning to issues of grounding and fundamentality, and applying them to firstorder disputes, it is important to periodically examine the foundations of this view. In this paper, we've examined one attempt to undermine the foundations of such theories. We have argued that deRosset's criticism of Priority Theory fails. The proposed constraint-(Det)-is not well-motivated, not a general constraint, and in fact is easily violated. Nevertheless, we have argued there is a more general problem, one that may not ultimately vitiate Priority Theory, but in any case will constrain how Priority Theory might be developed, as it surely must be.

\section{A Appendix: Further Formal Issues}

In our argument in $\S 4.5$ we urged a defeasible proposal: all things equal, we would expect a truthpreserving consequence relation that modeled generalizing from an arbitrary case will have (GoC). We recognize that there might be other ways of modeling this kind of reasoning. However, we contend that alternative ways of modeling this reasoning will not avoid the challenge we are trying to press here. We'll illustrate this point by considering a proposal due to an anonymous reviewer.

There is a familiar way of taking extensions of a consequence relation in mathematical logic. For example, if $\vdash$ is a consequence relation and $\Lambda$ is a set of sentences, we can define the extension $\vdash_{\Lambda}$ by

$$
\Gamma \vdash_{\Lambda} \psi \text { iff } \Gamma \cup \Lambda \vdash \psi
$$

Since $\vdash_{\Lambda}$ essentially treats the members of $\Lambda$ as tacit premises we'll call this a premise extension of $\vdash$. $\vdash_{\Lambda}$ is a truth-preserving consequence relation if $\vdash$ is, and it needn't have (GoC) in full generalityfor instance, if some constant $c$ occurred in $\Lambda$ then $c$ won't act like an arbitrary constant that can be universally generalized over. Nevertheless, $\vdash_{\Lambda}$ may still model generalizing from an arbitrary case: provided $\vdash$ itself had $(\mathrm{GoC})$, it is easy to see that for a given premise set $\Gamma$, the constants that can be universally generalized are just those that don't occur anywhere in $\Gamma$ or $\Lambda$.

Hence a premise extension like $\vdash_{\Lambda}$ shows how a truth-preserving consequence relation can model generalizing from arbitrary case without having $(\mathrm{GoC})$ in full generality. However, we maintain that if the consequence relation that figures in (Extended Grounding Explanation) is a relation like this that will still be enough to pose our challenge.

To see how, let's return to Priority Theory developed in terms of grounding (§4.5). Suppose the consequence relation $\Rightarrow$ we define as an extension the grounding relation, > , didn't have (GoC) in full generality but that it modeled generalizing from an arbitrary case by being an a premise extension of a consequence relation, $\Rightarrow^{\circ}$, which itself has (GoC). In particular, for a fixed set $\Lambda$, for any $\Delta$ and $\psi$,

$$
\Delta \Rightarrow \psi \text { if and only } \Delta \cup \Lambda \Rightarrow^{\circ} \psi
$$

Return now to the kind of example we have been discussing: let $a$ be nonfundamental, $\phi(a)$ be a non-total fact, and let $\Gamma$ be the explanantia of some explanation, $\phi(a)$ because $\Gamma$, where $a$ doesn't occur in $\Gamma$. The principle (Extended Grounding Explanation) will still hold for $\Rightarrow$, so $\Gamma \Rightarrow \phi(a)$. And, since $\Rightarrow$ is a premise extension of $\Rightarrow^{\circ}, \Gamma \cup \Lambda \Rightarrow^{\circ} \phi(a)$. The question now is whether $a$ appears in $\Lambda$ or not. If it does not, then, given that $\Rightarrow^{\circ}$ has $(\mathrm{GoC})$, a contradiction is derivable just as before. However, $a$ does occur in $\Lambda$, then we in a situation exactly parallel the case of Metaphysical Laws we discussed in $\S 4.4$. And what we had to say there applies equally well here. 


\section{References}

[1] Armstrong, David M. (2004). Truth and Truthmakers. Cambridge University Press.

[2] Anchinstein, Peter (1985). The Nature of Explanation. Oxford University Press. Oxford University Press.

[3] Audi, Paul (2012). 'A Clarification and Defense of the Notion of Grounding'. In Fabrice Correia and Benjamin Schnieder, eds., Grounding and Explanation. Cambridge University Press

[4] Beebee, Helen \& Dodd, Julian (eds.) (2005). Truthmakers: The Contemporary Debate. Clarendon.

[5] Bennett, Karen (2011). 'By Our Bootstraps' Philosophical Perspectives 25.1: 27-41.

[6] Bennett, Karen (2017). Making Things Up. Oxford University Press.

[7] Bliss, Ricki \& Trogdon, Kelly (2014). 'Metaphysical Grounding'. The Stanford Encyclopedia of Philosophy (Winter 2014 Edition), Edward N. Zalta (ed.), URL = $\langle$ http://plato.stanford.edu/archives/win2014/entries/grounding/ $\rangle$.

[8] Carnap, Rudolph (1950). Logical foundations of probability. University of Chicago Press

[9] Carnap, Rudolph (1966 [1995]). An Introduction to the Philosophy of Science. Ed. Martin Gardner. Dover.

[10] Chalmers, David J., Manley, David \& Wasserman, Ryan (eds.) (2009). Metametaphysics: New Essays on the Foundations of Ontology. Oxford University Press.

[11] Correia, Fabrice (2015). 'Logical Grounding and First-Degree Entailments'. Grazer Philosophische Studien. 91: 315

[12] Cleland, Carol E. (2011). 'Prediction and Explanation in Historical Natural Sciences'. British Journal for Philosophy of Science. 62.3: 551-82.

[13] deRosset, Louis (2010). 'Getting priority straight'. Philosophical Studies 149 (1):73 - 97.

[14] deRosset, Louis (2013). 'Grounding Explanations'. Philosopher's Imprint 13.7: 1-26.

[15] Enderton, Herbert B. (2001). A mathematical introduction to logic. 2nd ed. San Diego: Harcourt/Academic Press.

[16] Fefferman, Solomon (2008). 'Harmonious Logic: Craig's Interpolation Theorem and Its Descendants'. Synthese 164.3: 341-57

[17] Field, Hartry (1974). 'Quine and the correspondence theory'. Philosophical Review 83 (2):200228.

[18] Fisher, David. (Forthcoming) 'A conflict between van Inwagens metaontology and his ontology' Synthese

[19] Fisher, David. (Unpublished) 'A regress for the ground-theoretic explication of anti-realism'

[20] Fine, Kit (2001) 'The question of realism'. Philosopher's Imprint 1.2: 1-30 
[21] Fine, Kit (2012). 'A Guide to Ground'. In Fabrice Correia \& Benjamin Schnieder (eds.), Metaphysical Grounding. Cambridge University Press. pp. 37-80.

[22] Glazier, Martin (2016). 'Laws and the Completeness of the Fundamental'. In Reality Making (eds.), Reality Making. Oxford University Press. pp. 11-36.

[23] Hempel, Carl. (1965). Aspects of Scientific Explanation. The Free Press.

[24] Hempel, Carl (1966). Philosophy of Natural Science. Prentice-Hall.

[25] Hinman, Peter (2005). Fundamental of Mathematical Logic A. K. Peters.

[26] Kelly, Thomas (2008). 'Common sense as evidence: Against revisionary ontology and skepticism'. Midwest Studies in Philosophy 32 (1):53-78.

[27] Kim, Jaegwon (1979). 'Causality, Identity, and Supervenience in the Mind-Body Problem'. Midwest Studies in Philosophy. 4.1: 31-49.

[28] Lipton, Peter (2004). Inference to the Best Explanation. Routledge: Taylor and Francis Group.

[29] Mares, Edwin (2014). 'Relevance Logic'. The Stanford Encyclopedia of Philosophy (Spring 2014 Edition), Edward N. Zalta (ed.), URL = 〈https://plato.stanford.edu/archives/spr2014/entries/logic-relevance/〉.

[30] Marker, David (2002). Model Theory: An Introduction Springer Science + Business Media, LLC.

[31] Mendelson, Elliott (1979). Introduction to Mathematic Logic

[32] Merricks, Trenton (2007). Truth and Ontology. Oxford University Press.

[33] Mitchell, Sandra (2009). Unsimple Truth. University of Chicago Press.

[34] O'Leary-Hawthorne, John \& Michael, Michaelis (1996). 'Compatibilist semantics in metaphysics: A case study'. Australasian Journal of Philosophy 74 (1):117 - 134.

[35] O'Connor, Timothy \& Hong Yu Wong (2005). 'The Metaphysics of Emergence'. Nô̂s 39 (4): 658-678.

[36] Quine, W. V. (1948) 'On What There Is'. Review of Metaphysics. 2.1: 21-38.

[37] Quine, W. V. (1960). Word and Object. MIT Press.

[38] Railton, Peter (1978). 'A Deductive-Nomological Model of Probabilistic Explanation'. Philosophy of Science 45.2: 206-26.

[39] Rodriguez-Pereyra, Gonzalo (2005). 'Why Truthmakers'. In H. Beebee \& J. Dodd (eds.), Truthmakers: the contemporary debate. Oxford University Press 17-31.

[40] Roski, Stefan \& Antje Rumberg (2016) 'Simplicity and Economy in Bolzano's Theory of Grounding'. Journal of the History of Philosophy, 54.3: 469-496

[41] Roski, Stefan (2017). Bolzano's Conception of Grounding Vittorio Klostermann

[42] Ruben, David-Hill (1990). 'Singular Explanations in Social Sciences'. Midwest Studies in Philosophy. XV: 130-149. 
[43] Russell, Gillian \& Greg Restall (2010) 'Barriers to Implication' In Charles Pigden (ed.), Hume on Is and Ought. Palgrave MacMillan.

[44] Salmon, Wesley (1989). Four Decades of Scientific Explanation. University of Pittsburg Press.

[45] Schaffer, Jonathan (2009). 'On what grounds what'. In David Manley, David J. Chalmers \& Ryan Wasserman (eds.), Metametaphysics: New Essays on the Foundations of Ontology. Oxford University Press 347-383.

[46] Schaffer, Jonathan (2010). 'The least discerning and most promiscuous truthmaker'. Philosophical Quarterly 60 (239):307 - 324.

[47] Scrivens, Michael (1959). 'Explanation and Prediction in Evolutionary Theory'. Science. 130.3374: 477-82.

[48] Shoenfield, Joeseph R (1967). Mathematical Logic Reading, Mass., Addison-Wesley Pub. Co.

[49] Skow, Bradford (2016). Reasons Why Oxford University Press.

[50] Sider, Theodore (2011). Writing the Book of the World. Oxford University Press.

[51] Sider, Theodore (Forthcoming) 'Ground Grounded'. Philosophical Studies

[52] Skiles, Alexander (2015). 'Against Grounding Necessitarianism'. Erkenntnis 80: 717-51.

[53] Tarski, Alfred (1983). 'The Concept of Truth in Formalized Languages'. in J. Corcoran ed. Logic, Semantics, Metamathematics Indianapolis: Hackett.

[54] Trogdon, Kelly (2018). 'Grounding-Mechanical Explanation'. Philosophical Studies 75 (6):1289-1309

[55] van Fraassen, Bas (1980). The Scientific Image. Clarendon Press.

[56] van Inwagen, Peter (1990). Material Beings. Cornell University Press.

[57] von Solodkoff, Tatjana (2012). 'Straightening priority out'. Philosophical Studies 161 (3):391401.

[58] Wallace, Meg (2011a). 'Composition as Identity, Part 1.' Philosophical Compass. 6.11: 804-816.

[59] wallace, Meg (2011b). 'Composition as Identity, Part 2.' Philosophical Compass. 6.11: 817-827.

[60] Wilsch, Tobias (2016). 'The Deductive-Nomological Account of Metaphysical Explanation'. Australasian Journal of Philosophy. 94.1: 1-23.

[61] Wittgenstein, Ludwig (1922). Tractatus Logico-Philosophicus. 